\title{
A Unified Geometrical Framework for Face-Centered Icosahedral Approximants in Al-Pd-TM (TM = Transition Metal) Systems
}

\author{
Nobuhisa Fujita $^{1, * 1}$ and Makoto Ogashiwa ${ }^{2, * 2}$ \\ ${ }^{1}$ Institute of Multidisciplinary Research for Advanced Materials, Tohoku University, Sendai 980-8577, Japan \\ ${ }^{2}$ Department of Materials Processing, Graduate School of Engineering, Tohoku University, Sendai 980-8579, Japan
}

\begin{abstract}
The complex atomic structure of a high-order approximant to face-centered icosahedral quasicrystal in Al-Pd-TM (TM = transition metal) systems can be deconvoluted into two kinds of atomic clusters pinned at the vertices of a tiling composed of four basic polyhedra, called the canonical cells. As a result, thousands of atoms per unit cell can be registered in fifteen orbits associated with vertices, edges, faces, and cells of the relevant canonical-cell tiling. This geometrical framework facilitates a rational guess of an atomic jungle in an unknown approximant structure, could an underlying tiling be postulated. A novel approximant phase in the Al-Pd-(Mo-Fe) system is discussed within the present framework. [doi:10.2320/matertrans.MT-MB2020007]
\end{abstract}

(Received September 1, 2020; Accepted October 27, 2020; Published December 4, 2020)

Keywords: icosahedral quasicrystals, approximants, X-ray crystallography, pseudo-Mackay cluster, mini-Bergman cluster, canonical cell tiling, atomic decoration models

\section{Introduction}

Quasicrystals $(\mathrm{QCs})^{1)}$ have been capturing abiding interests across wide areas of science and engineering. Much effort has been invested into the synthesis of new compounds, ${ }^{2,3)}$ elucidation of their extraordinary structure ${ }^{4,5)}$ and unique physical properties. ${ }^{6}$ ) Among others, thermodynamically stable icosahedral QCs in Al based alloys ${ }^{7-11)}$ have been attracting extensive attention due to their low production costs and potential applications that can be industrially feasible. ${ }^{12-14)}$

As quoted by Bak in the famous article entitled "Icosahedral Crystals: Where are the Atoms?", ${ }^{15)}$ it has never been a simple task to determine the atomic structure of real icosahedral QCs. A three-dimensional (3D) atomic arrangement in an icosahedral QC is formally obtained as a $3 \mathrm{D}$ section of a hypothetical periodic structure, so called a hyper-crystal, ${ }^{16)}$ in a $6 \mathrm{D}$ hyperspace. There are three potential $6 \mathrm{D}$ Bravais lattices for the hyper-crystal whose point symmetry group is isomorphic to the icosahedral point symmetry group, $I_{\mathrm{h}}$, in three dimensions $;{ }^{17-19)}$ namely, the simple-icosahedral (SI), face-centered icosahedral (FCI), and body-centered icosahedral (BCI) lattices. To date, real-life icosahedral QCs are classified exclusively into the SI and FCI Bravais classes. The hyper-crystal is a periodic arrangement of well-defined geometrical objects extending along the 3D orthogonal complements (called the perpendicular space) to the $3 \mathrm{D}$ physical space. In the literature, the latter objects are called atomic surfaces or, equivalently, occupation domains, acceptance domains, etc. ${ }^{16)}$

For investigating the atomic structure of an icosahedral $\mathrm{QC}$, it has been a common practice to extrapolate the structural knowledge of approximants (APs), ${ }^{20)}$ which are crystalline compounds with a significant structural complexity and which exist in a compositional proximity to the QC. APs are usually composed of atomic clusters templated by a few concentric polyhedral shells with icosahedral symme-

${ }^{* 1}$ Corresponding author, E-mail: nobuhisa.fujita.a4@tohoku.ac.jp

${ }^{* 2}$ Graduate Student, Tohoku University try, ${ }^{2,21)}$ implying that the QC is composed of similar clusters. The periodic packing of clusters in an AP corresponds to a periodic approximation to a quasiperiodic packing of clusters in the QC.

Within the hyperspace formalism, the quasiperiodic packing of clusters is described using an appropriate atomic surface that generates the cluster centers. However, such an atomic surface cannot be determined solely from diffraction data in sharp contrast to that in an ordinary periodic crystal all the atomic positions can be determined $a b$ initio using single-crystal methods. Here, an empirical choice of the atomic surface is imperative prior to replicating the cluster shells around each cluster centers., ${ }^{4,5,22-24)}$ It is therefore vital to have structural knowledge from high-order APs, which closely mimic the QC with respect to the packing of clusters, for a reliable modelling of the QC structure. To date a limited number of such APs with huge unit cells have been known, and besides it is in itself a challenging task to solve their atomic structures.

In this paper, we present an account on the atomic structure of high-order APs to FCI type QCs in Al-Pd-TM (TM= transition metal) systems. In particular, we make use of the canonical-cell tiling $(\mathrm{CCT}),{ }^{25)}$ which is a tiling framework with four basic polyhedra called the canonical cells and which can describe the packing of clusters in a wide range of APs to icosahedral QCs ranging from relatively simple to extremely complex ones. ${ }^{26)}$ Section 2 reviews some recent advances in the structural understanding of FCI type QCs and their APs in Al-Pd-TM systems, with a focus on the recent discovery of a high-order cubic AP in the $\mathrm{Al}-\mathrm{Pd}-(\mathrm{Cr}-\mathrm{Fe})$ system $^{27)}$ exhibiting two kinds of icosahedral clusters. The central atoms of the constituent clusters are pinned at the vertices of a CCT corresponding to a $2 \times 2 \times 2$ superstructure of the $3 / 2$ cubic packing, ${ }^{25)}$ whereas all the other atoms belong to the cluster shells. We note that this description applies also to a few additional compounds with the same ${ }^{28)}$ and different underlying CCTs. ${ }^{29,30)}$ An instructive account on the tiling framework is provided in Sec. 3 to give a sense of a wider applicability of the present approach to a general class of APs and FCI type QCs as well. 
In Sec. 4, basic rules for atomically decorating a CCT are derived from the prototype structure. ${ }^{27)}$ Fifteen distinct orbits with respect to the proper symmetries of vertices, edges, faces and cells are identified and correlated with the refined atomic structure. This facilitates a rational guess of an unknown atomic jungle of a novel AP compound. Finally, in Sec. 5, the present framework is applied to a novel AP in the $\mathrm{Al}-\mathrm{Pd}-(\mathrm{Mo}-\mathrm{Fe})$ system to highlight the general applicability of the present framework to a wider class of complex APs. Despite that no decisive structure solution has been given by ordinary X-ray crystallography, a reasonable structure model is readily constructed from a postulated CCT that underlies the compound. The validity of such a model could be verified by comparing computed and observed high-resolution X-ray diffraction patterns.

\section{High-Order Cubic Approximants in Al-Pd-TM Systems}

Soon after the discovery of stable FCI type QCs in Albased alloys, ${ }^{7-9)}$ Katz and Gratias proposed a geometrical model of the atomic structure based on the hyperspace formalism. ${ }^{31,32)}$ It was noticed that their model contained two kinds of clusters, one with Mackay type and the other with Bergman type cluster shells, which were centered at vertex and body centered positions, respectively. Meanwhile, Elser ${ }^{33)}$ proposed another structure model, in which similar two kinds of small clusters named 'Mackay volume' and 'Bergman dodecahedron' alternately decorate the vertices of tilings composed of Ammann rhombohedra, or more precisely a random tiling ensemble. Here, icosahedral symmetry applies not to individual structures but to the ensemble so as to rationalize the entropic stabilization of the icosahedral QCs. Bear in mind that the two rhombohedra are also known as the prototiles of the three-dimensional Penrose tiling. ${ }^{34-37)}$

For more than a decade, however, there had been no experimental evidence that would have directly confirmed the two kinds of small clusters as the proper building units of the FCI type QCs and their APs. Instead, the single crystal refinements of the $1 / 1$ and $2 / 1$ cubic APs in the Al-Pd-MnSi system due to Sugiyama et al ${ }^{38,39)}$ revealed much larger cluster units, which were subsequently incorporated by Yamamoto et al. ${ }^{5)}$ into a hyperspace model of the FCI type $\mathrm{QC}$ in the Al-Pd-Mn system. Elser's small clusters could not be accepted if Sugiyama's large clusters were considered relevant, and vice versa. In fact, the discrepancy might be attributed to the low quality of the materials that were taken from an as synthesized ingot after arc melting. ${ }^{38,39)}$ A reliable structural refinement might have been hindered by a significant amount of stacking disorder in the samples.

\subsection{Prototype compound with two kinds of clusters}

A real-life compound that exhibits the two small clusters used by Elser ${ }^{33)}$ as the building units was only recently discovered in the $\mathrm{Al}-\mathrm{Pd}-(\mathrm{Cr}-\mathrm{Fe})$ system, with a composition of $\mathrm{Al}_{\sim 69} \mathrm{Pd}_{\sim 22} \mathrm{Cr}_{\sim 2} \mathrm{Fe}_{\sim 7} .{ }^{27)}$ The complex crystal structure containing as many as 264 small clusters amounting 4320 atoms per unit cell with the space group $P a \overline{3}$ and a lattice constant of $40.5 \AA$ was determined using single-crystal X-ray
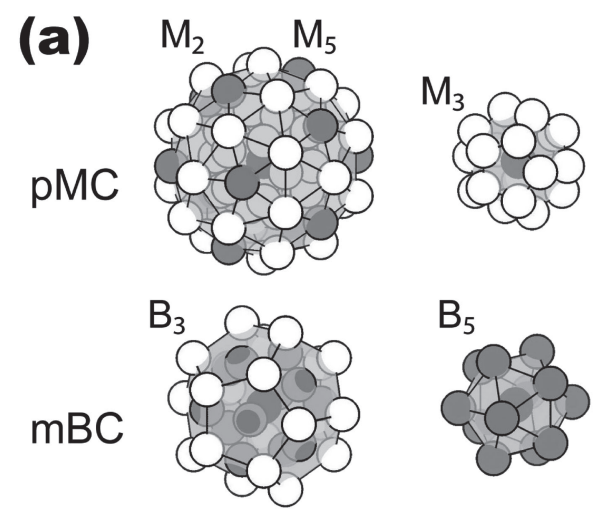

$\mathrm{B}_{0}$

(b)

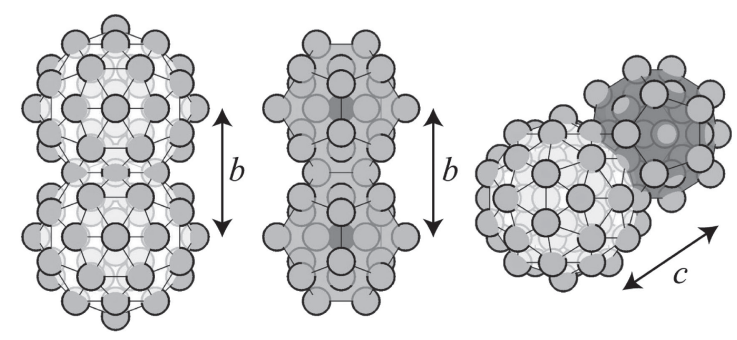

Fig. 1 (a) Geometrical templates of $\mathrm{pMC}$ and $\mathrm{mBC}$, both consisting of the central atom and two concentric shells with icosahedral symmetry. The outer shell of pMC can be decomposed further into two subshells. Shells are mutually distinguished by symbols in which the proper rotations at each site is represented as a subscript. (b) Three kinds of linkages between the cluster units. Adjacent clusters of the same kind are connected through a $b$ linkage, while ones of the opposite kinds are connected through a $c$ linkage.

diffraction (SCXRD). A single phase polycrystalline sample of this compound was synthesized by annealing an arcmelted alloy ingot with the nominal composition at $1123 \mathrm{~K}$, while a single grained sample was obtained via slow cooling method (at a rate of $10 \mathrm{~K} /$ hour) following the melting of the ingot at $1433 \mathrm{~K}$.

The crystal structure is composed of two kinds of cluster units being as small as $\sim 8 \AA$ in diameter. These are called pseudo-Mackay clusters (pMCs) and mini-Bergman clusters (mBCs), which are templated by a central atom and two concentric polyhedral shells with icosahedral symmetry (Fig. 1(a)). ${ }^{27)}$ The two shells of a pMC (except for its center) accommodate vacancies to avoid too short inter-atomic distances. On the other hand, the two shells of an $\mathrm{mBC}$ are fully occupied. In general, an uneven distribution of elements or vacancies deprives an individual cluster shell of an exact icosahedral symmetry.

One finds that each cluster can be connected to its adjacent neighbors through three types of inter-cluster linkages: (i) $\mathrm{mBC}-\mathrm{mBC}$, (ii) $\mathrm{pMC}-\mathrm{pMC}$, and (iii) $\mathrm{mBC}-\mathrm{pMC}$. (Figure 1(b)). Either (i) or (ii) is parallel to a 2-fold axis with a length (i.e., the distance between the two cluster centers) of $\sim 7.7 \AA$ ( $b$ linkage). (iii) is parallel to a 3 -fold axis with a length of $\sim 6.7 \AA$ ( $c$ linkage). The ideal ratio between the two lengths is $b: c=2: \sqrt{3}$.

Atoms are generally shared between adjacent clusters. For instance, all the inner shell atoms of a pMC are shared with 
the outer shells of the adjacent mBCs connected through $c$ linkages. Similarly, some of the inner shell atoms of an $\mathrm{mBC}$ are shared with the outer shells of the adjacent pMCs. The configuration of atoms (or that of vacancies) in the inner shell of a pMC is particularly dependent on the configuration of the adjacent clusters. ${ }^{27)}$

\subsection{Structural description with CCT}

The prototype structure has brought new insights into the structural investigation of the FCI type icosahedral QCs in Al-based systems. ${ }^{40}$ In the hyperspace formalism, the $6 \mathrm{D}$ lattice points corresponding to the two ends of a $b$ linkage have the same parity (even-even or odd-odd), while the 6D lattice points corresponding to the two ends of a $c$ linkage have opposite parities (even-odd or odd-even). ${ }^{27)}$ Apparently, assigning two different kinds of clusters to even and odd vertices induces an FCI type ordering in the structure.

It is also important to note that the network of $b$ and $c$ linkages identified in the prototype compound presents the edge network of a CCT that can be designated as a $2 \times 2 \times 2$ superstructure of the $3 / 2$ cubic packing. ${ }^{25)}$ Hence, the compound is hereafter denoted as the $(2 \times 2 \times 2)$ cubic 3/2 AP.

The present description is not compatible with Elser's model, ${ }^{33)}$ in which an FCI type ordering is induced by replicating two kinds of clusters at opposite ends of each rhombohedral edge (parallel to a 5-fold axis, also termed $\tau^{2} a$ linkage).

The present observation assures that the atomic structure of the $(2 \times 2 \times 2)$ cubic $3 / 2$ AP can be described as an atomic decoration of the CCT. One may notice here that each of the four basic cells, namely, $A, B, C$ and $D$ cells, shown in Fig. 2(a) can be decorated in two distinct ways depending on the assignment of even and odd parities to its corners. In Sec. 4 , an atomic decoration scheme for CCTs will be introduced.

\section{CCT Framework}

One might feel uneasy trying to grasp the geometry of the CCT. Indeed, the shapes of the four cells might look too intricate to remember for those even new to the icosahedral tiling composed of Ammann rhombohedra. ${ }^{34-36)}$ Therefore, it should be useful here to give a brief geometrical introduction to the CCT framework ${ }^{25)}$ along with selected examples, from simple to complex ones, showcasing what are the most relevant to real-life APs to icosahedral QCs.

\subsection{Preliminary remarks on the geometry}

The $b$ and $c$ linkages correspond to the edges of the canonical cells. There are three kinds of face, namely, an isosceles triangle bounded by $b c c$ edges ( $X$ face), an equilateral triangle bounded by $b b b$ edges ( $Y$ face), and a rectangle bounded by $b c b c$ edges ( $Z$ face). For an SI-type structure, all the vertices are decorated with the same kind of cluster. An FCI type structure on the other hand distinguishes the two kinds of vertices, even and odd, to be decorated with pMCs and $\mathrm{mBCs}$, respectively. Here, the parity of each vertex is that of the sum of six indices which define the coordinate of the vertex as a linear combination of the icosahedral basis vectors. (a)
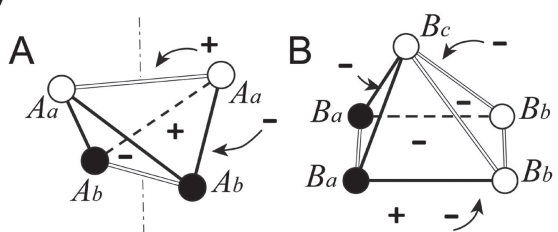

$b$
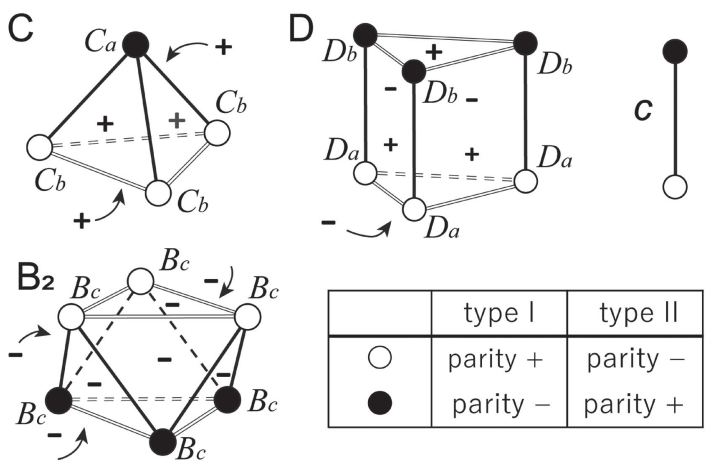

(b)

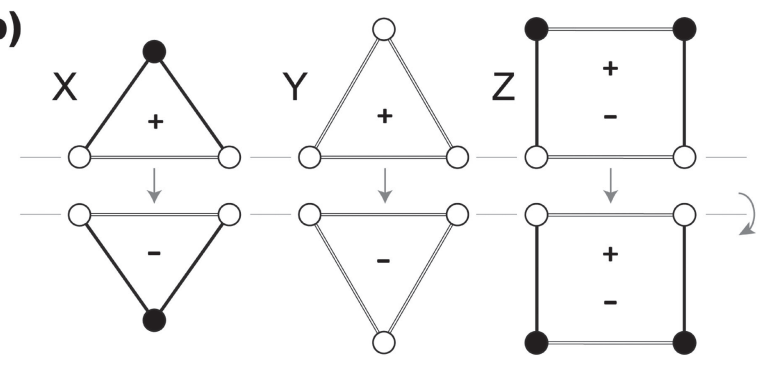

Fig. 2 Geometrical objects in the CCT framework. (a) The four fundamental cells (namely, A, B, C, and D cells) along with a trigonal anti-prism composed of two $B$ cells $\left(B_{2}\right.$ cell). The symmetrically inequivalent corners are distinguished by the corner symbols (e.g., $A_{a}$, $C_{b}$, etc.) due to Henley. ${ }^{25}$ ) White and black discs signify the distinct parities of the vertices. (b) The three kinds of faces (namely, X, Y, and Z faces) and their flipped sides with respect to a two-fold axis. The + and - signs on the faces mark their inequivalent sides. The two sides of a $\mathrm{Z}$ face are equivalent apart from that the parities of the vertices are opposite. There are two inequivalent ways to assign the parities to the four cells (tabulated as type I and type II) as well as to X and Y faces.

In order to tile the space without gap, any CCT must conform to the face-on-face matching constraints. Each $Y$ face, for instance, can be shared by a pair of $B C, B D$, or $C D$ cells. The two sides of a $\mathrm{Y}$ face are symmetrically inequivalent, as symbolically represented in Fig. 2(b) by + and - charges on the surface. The sharing of the $Y$ faces enforces the charge neutrality throughout the structure, which ensures that the number frequencies of $B$ and $C$ cells in CCTs are always identical. ${ }^{25)}$

It is known that 32 distinct types of local vertex environments are allowed in CCTs. ${ }^{25)}$ One that is most frequently seen is depicted in Fig. 3: the vertex is connected through $6 \quad b$ linkages and $7 c$ linkages to its adjacent neighbors, while it is shared by corners of $12 \mathrm{~A}$ cells, $3 \mathrm{~B}$ cells and $4 C$ cells that altogether fill up the entire solid angle, $4 \pi$. Following Henley, ${ }^{25)}$ we use the symbol $(67)_{333}$ for representing this type of vertex environment. All the 32 types of vertex environments are tabulated in the literature. ${ }^{25,41)}$

\subsection{CCTs: From simple to complex}

The simplest CCT also known as the $1 / 1$ cubic packing ${ }^{25)}$ 

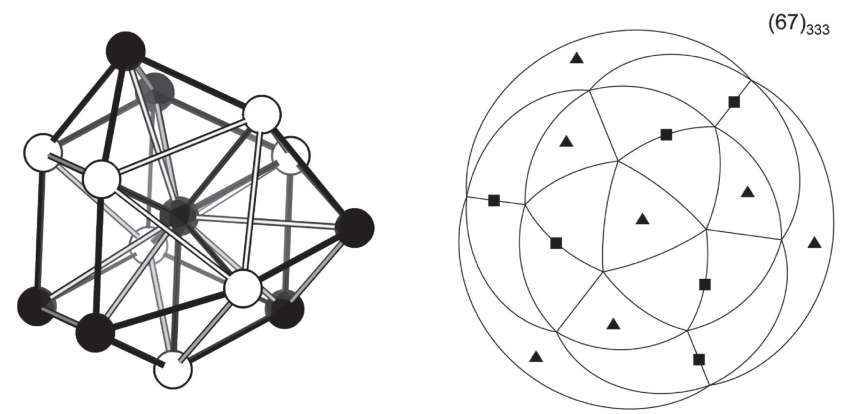

Fig. 3 Left panel: The most frequent type of vertex environment, (67) ${ }_{333}$ Right panel: A planer diagram ${ }^{41)}$ representing the relative orientations of the $6 b$ and $7 c$ linkages with respect to projected icosahedral frames, where the 3 -fold axis is in the center. (a)

(b)

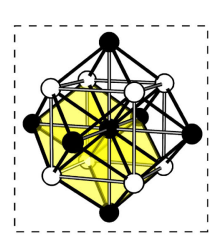

(d)

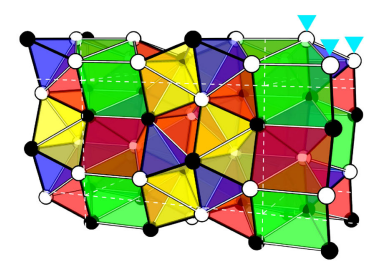

(e)

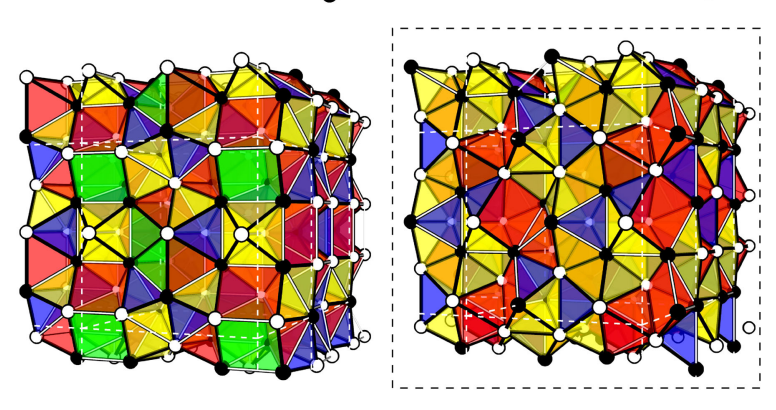

(c)

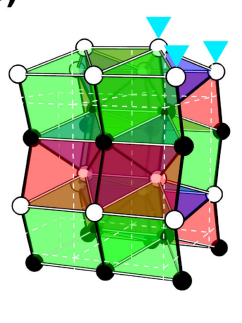

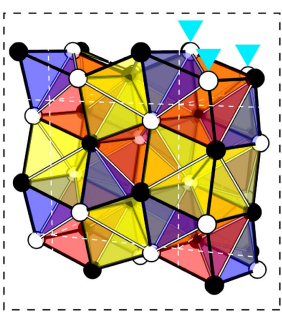

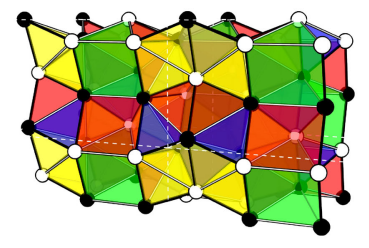

Fig. 4 Important examples of CCTs, where only the ones with cubic symmetry are surrounded by dashed rectangles. $A, B, C$, and $D$ cells are colored in yellow, red, blue, and green, respectively. (a) The $1 / 1$ cubic packing with a BCC arrangement of the vertices. Only an $A_{6}$ rhombohedron is highlighted in color. (b) The $2 / 1$ cubic packing exhibiting an alternation of $A_{6}$ and $B_{2} C_{2}$ rhombohedra. (c) The $(2 / 1)^{2}(1 / 1)$ orthorhombic packing consisting of $B, C$, and $D$ cells. (d) The $(2 / 1)^{2}(3 / 2)$ orthorhombic packing (right) and the $(2 / 1)^{2}(3 / 2)$ monoclinic packing (left). (e) The $3 / 2$ cubic packing (right) and its rhombohedral variant (left).

is composed only of $A$ cells. As shown in Fig. 4(a), the vertices are arranged on a body-centered cubic (BCC) lattice, where the lattice parameter is $b$, i.e. the length of $b$ linkage. The BCC primitive unit cell is a rhombohedron comprising six $A$ cells, or a $A_{6}$ rhombohedron. The second simplest CCT with a cubic unit cell is the $2 / 1$ cubic packing, ${ }^{25)}$ which exhibits an alternation of $A_{6}$ and $B_{2} C_{2}$ rhombohedra, the latter of which comprises doublets of $B$ and $C$ cells (Fig. 4(b)). The lattice parameter of the $2 / 1$ cubic packing is $\tau b$, where $\tau=$ $(1+\sqrt{5}) / 2$ is the golden mean. Figure 4(c) shows another simple CCT known as the $(2 / 1)^{2}(1 / 1)$ orthorhombic packing, ${ }^{25)}$ composed only of $B, C$, and $D$ cells, with the lattice parameters being $\tau b, \tau b$, and $b$. Note in the figure that the third axis (with period $b$ ) is in the rightward direction, exposing a puckered surface (with $X$ and $Z$ faces) normal to the axis.

We point out that the same kind of puckered surface can be exposed by cleaving the $2 / 1$ cubic packing, such that $A_{6}$ and $B_{2} C_{2}$ rhombohedra are cut in half at the surface. This can be seen if the vertical zig-zag arrays of edges marked with lightblue arrowheads in Fig. 4(b) are exposed on the surface. Then, if we translate the right side of the cleaved surface bit away from the rest, we can create a crack with a thickness of $b$. Now the crack can be filled perfectly with a single layer of the $(2 / 1)^{2}(1 / 1)$ orthorhombic packing, which introduces a stacking fault layer into the $2 / 1$ cubic structure. Notice that similar stacking faults can be introduced by arbitrary number of times in the same direction. For instance, a stacking fault can be introduced within each and every period along the third axis. This leads to another periodic CCT with an orthorhombic unit cell with the lattice parameters being $\tau b$, $\tau b$, and $\tau^{2} b(=\tau b+b)$ (Fig. 4(d), left). This is in fact known as the $(2 / 1)^{2}(3 / 2)$ monoclinic packing structure. ${ }^{25)}$ Moreover, we can readily realize the Fibonacci sequence of thick $(\tau b)$ and thin $(b)$ layers to obtain a quasi-1D quasiperiodic CCT, which might provide a potential interpretation of 1D QCs reported in the $\mathrm{Al}-\mathrm{Pd}-\mathrm{Fe}$ system. ${ }^{42,43)}$

Now, a question arises as to the possibility that similar stacking faults could be introduced simultaneously along the 3 independent orthogonal axes. In fact, a parallel set of stacking fault layers similar to that shown in the left panel of Fig. 4(d) can be inserted simultaneously in all 3 orthogonal axes of the $2 / 1$ cubic structure subject to that extra 6 vertices are manually added at every intersection of 3 mutually orthogonal stacking fault layers. The left panel of Fig. 4(e) depicts the resulting structure that has a cubic unit cell with a lattice parameter of $\tau^{2} b$, and is in fact a rhombohedral variant (space group, $R \overline{3}$, if even and odd vertices are not distinguished) of the $3 / 2$ cubic packing ${ }^{25)}$ (Fig. 4(e), right).

Unfortunately, it is unlikely that a similar process of introducing stacking faults could be applied indefinitely along three independent directions. At present, the only way that we know of to obtain a CCT that is genuinely quasiperiodic in three dimensions uses inflation. ${ }^{41)}$

\section{CCT Decoration Scheme for Modeling the Structure}

A CCT decoration scheme for modeling the atomic structure of APs to FCI type QCs is defined by a set of orbits with respect to the proper symmetries of vertices (point group, $\overline{5} \overline{3} \mathrm{~m})$, edges $(b: m m m, c: 3 m)$, faces $(X: m, Y: 3 m$, $Z: m)$, cells $(A: 2 m m, B: m, C: 3 m, D: 3 m)$, or any cluster of the latter (e.g., $B_{2}: 3 m$ ). Note that some of the proper symmetries are lower than those reported in the literature ${ }^{25)}$ because of the distinction between even and odd vertices to take into account the FCI type ordering. The atomic positions in the structure should be uniquely registered in these orbits, which on the contrary should cover all the atomic positions without overlapping. ${ }^{44)}$ Figure 5(a) shows a simple illustrative example of modeling the atomic structure of a decagonal QC by decorating the rhombic Penrose tiling with 
(a)

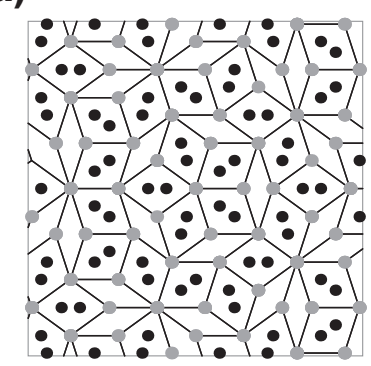

(c)

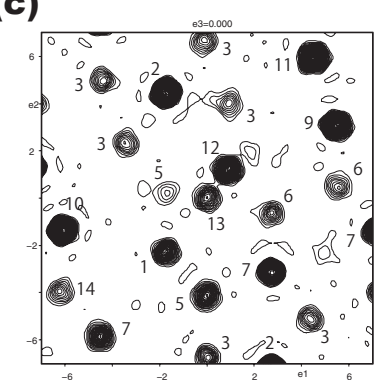

(b)

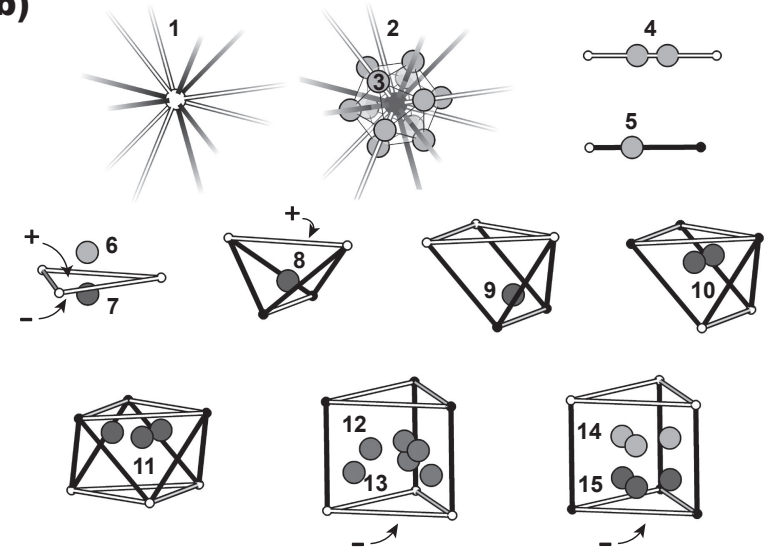

Fig. 5 (a) A decagonal QC model derived from the rhombic Penrose tiling by introducing two kinds of decoration orbits: a single atom (species $\mathrm{A}$, gray) for each vertex and a pair of atoms (species B, black) for each fat rhombus. (b) The fifteen orbits in the present decoration scheme for CCTs. The white and black colors of the vertices correspond to even and odd vertices, respectively. Except for the cluster centers, atomic sites are represented either as light spheres (for $\mathrm{Al}$ ) or dark spheres (for TM including Pd). (c) A 2D section of the electron charge density map resulting from the Fourier synthesis of the prototype Al-Pd- $(\mathrm{Cr}-\mathrm{Fe})$ compound. ${ }^{27)}$ The plane cut through the center of a type I D cell, $D_{+++---}$.

two kinds of orbit: a single atom (species A) at each vertex, and a pair of atoms (species B) on the long diagonal of each fat rhombus.

In order to register all the atomic positions in the refined atomic structure of the $(2 \times 2 \times 2)$ cubic $3 / 2 \mathrm{AP}$, it is practically sufficient to introduce fifteen orbits which are depicted in Fig. 5(b). In the following, a specific symbol is defined for each of these decoration orbits, where the superscript denotes the element that has the largest occupancy in the refined structure model (see Table 1). The multiplicity, $m$, of each orbit is given in the parentheses at the end of each line as $(\times m)$.

(1) $n_{+}^{F e}:$ a site associated with every even vertex, corresponding to the central atom $\left(\mathrm{M}_{0}\right)$ of the relevant pMC. $(\times 1)$

(2) $n_{-}^{P d}:$ a site associated with every odd vertex, corresponding to the central atom $\left(\mathrm{B}_{0}\right)$ of the relevant $\mathrm{mBC} .(\times 1)$

(3) $n_{-}^{A l}$ : an icosahedron associated with every odd vertex, corresponding to the inner icosahedral shell $\left(\mathrm{B}_{5}\right)$ of the relevant $\mathrm{mBC} .(\times 12)$

(4) $b_{++}^{A l}$ : a pair of sites associated with every $b_{++}$linkage, corresponding to a pair of split sites belonging to the relevant $\mathrm{M}_{2}$ shells. $(\times 2)$
(5) $c_{+-}^{A l}$ : a single site associated with every $c$ linkage, corresponding to the intersection of the relevant $\mathrm{M}_{3}$ and $\mathrm{B}_{3}$ shells. $(\times 1)$

(6) $Y_{+++}^{A l}$ : a single site on the normal centroidal line of every $\mathrm{Y}$ face spanned by three even vertices, corresponding to the intersection of the relevant three $\mathrm{M}_{2}$ shells. $(\times 1)$

(7) $Y_{+++}^{F e}$ : a single site on the normal centroidal line of every $\mathrm{Y}$ face spanned by three even vertices, corresponding to the intersection of the relevant three $\mathrm{M}_{5}$ shells. $(\times 1)$

(8) $A_{++--}^{P d}$ : a single site near the centroid of every A cell spanned by two even and two odd vertices in the specified order (type I), corresponding to the intersection of the relevant $\mathrm{M}_{5}, \mathrm{M}_{5}, \mathrm{~B}_{3}$, and $\mathrm{B}_{3}$ shells. $(\times 1)$

(9) $B_{--+++}^{P d}$ : a single site inside every non-paired $\mathrm{B}$ cell spanned by three even and two odd vertices (type I), corresponding to the intersection of $\mathrm{M}_{5}, \mathrm{~B}_{3}$, and $\mathrm{B}_{3}$ shells associated with an $\mathrm{X}$ face. $(\times 1)$

(10) $B_{++---}^{P d}$ : a pair of sites inside every non-paired $\mathrm{B}$ cell spanned by two even and three odd vertices (type II), corresponding to the intersection of $\mathrm{M}_{5}, \mathrm{~B}_{3}$, and $\mathrm{B}_{3}$ shells associated with an $\mathrm{X}$ face. $(\times 2)$

(11) $B_{2}^{P d}$ : a triad of sites inside every paired $\mathrm{B}$ cells spanned by three even and three odd vertices, corresponding to the intersection of $\mathrm{M}_{5}, \mathrm{~B}_{3}$, and $\mathrm{B}_{3}$ shells associated with an $\mathrm{X}$ face. $(\times 3)$

(12) $D_{+++---}^{P d 1}:$ a triad of sites inside every D cell spanned by even and odd vertices in the specified order (type I), corresponding to the intersection of M5 and B3 shells associated with a $c$ linkage. $(\times 3)$

(13) $D_{+++-_{--}}^{P d 2}$ : a triad of sites inside every D cell spanned by even and odd vertices in the specified order (type I), corresponding to the split sites for $D_{+++---.}^{P d 1}(\times 3)$

(14) $D_{---+++}^{A l}$ : a triad of sites inside every D cell spanned by even and odd vertices in the specified order (type II), corresponding to the intersection of two $\mathrm{M}_{2}$ shells associated with a $b_{++}$linkage. $(\times 3)$

(15) $D_{---+++}^{P d}$ : a triad of sites inside every D cell spanned by even and odd vertices in the specified order (type II), corresponding to the intersection of two $\mathrm{B}_{3}$ shells associated with a $b_{--}$linkage. $(\times 3)$

The distance deviations of the refined atomic positions of the $(2 \times 2 \times 2)$ cubic $3 / 2$ AP in the Al-Pd- $(\mathrm{Cr}-\mathrm{Fe})$ system from the corresponding ideal decoration sites are compared in Table 1. The root-mean-squared deviation is less than 0.2 angstrom except for $b_{++}^{A l}, c_{+-}^{A l}$, and $D_{+++---}^{P d 1}$ (underlined). Note that both $b_{++}^{A l}$ and $c_{+-}^{A l}$ register $\mathrm{Al}$ atoms which are encaged by the outer shell $\left(\mathrm{M}_{5}\right.$ and $\left.\mathrm{M}_{2}\right)$ of a pMC, where positional disorder is promoted due to a large number of potential atomic sites with only fractional occupancies. Meanwhile, one of the three Pd atoms in the $D_{+++---}^{P d 1}$ triad is statistically pushed towards the center of a pMC to take the split position in $D_{+++--}^{P d 2}$, as it were, to homogenize the atomic mass distribution in favor of entropic stability.

In Table 1, the site occupation statistics of the four elements for all the fifteen orbits are also given, demonstrating that the atomic species depend strongly on the local geometrical environments of the individual decoration sites. 
Table 1 Atomic-site statistics in the $(2 \times 2 \times 2)$ cubic $3 / 2$ AP in the Al-Pd- $(\mathrm{Cr}-\mathrm{Fe})$ system with respect to the fifteen decoration orbits.

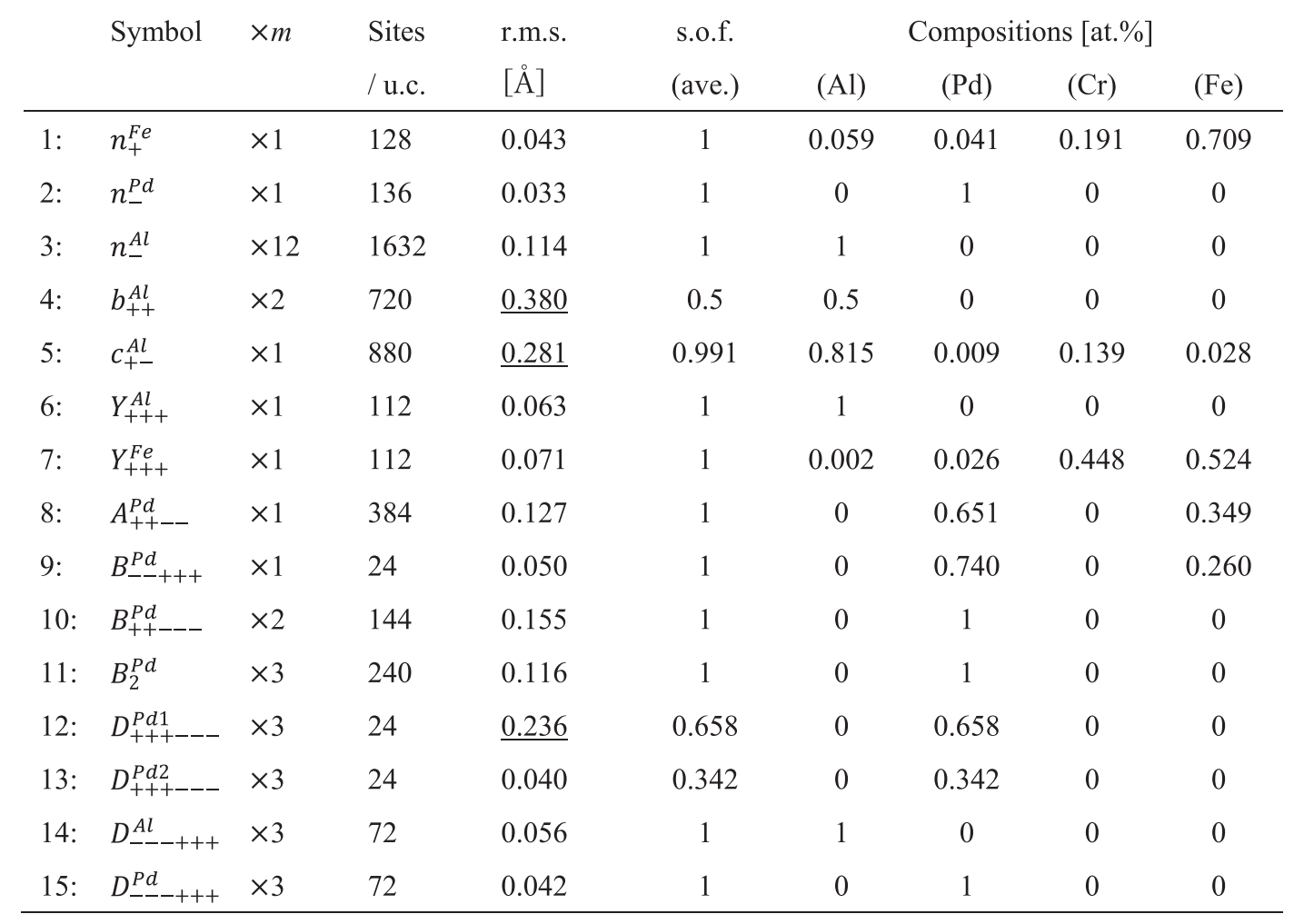

\section{Structural Variants}

The present geometrical framework applies not only to the $(2 \times 2 \times 2)$ cubic $3 / 2$ AP in the Al-Pd- $(\mathrm{Cr}-\mathrm{Fe})$ system, ${ }^{27)}$ but also to a few other complex APs reported to date, including the $\mathrm{P}_{40}-\mathrm{AlPdRu},{ }^{28)} \mathrm{F}-\mathrm{AlCoPdGe},{ }^{29)}$ and R$\mathrm{AlCoPd}^{30)}$ compounds. All the latter can be deconvoluted into pMCs and $\mathrm{mBCs}$, which are centered at the vertices of CCTs. The atoms are in principle registered in the fifteen orbits, each demonstrating a characteristic on-site chemistry. In particular, $\mathrm{P}_{40}$-AlPdRu is isostructural to the $(2 \times 2 \times 2)$ cubic $3 / 2$ AP. $^{28)}$

We recently conducted a systematic search for similar structures in the $\mathrm{Al}-\mathrm{Pd}-(\mathrm{Mo}-\mathrm{Fe})$ system, which replaces $\mathrm{Cr}$ in the prototype compound with Mo in the same group. The search culminated in two structurally distinct AP phases with virtually an identical composition of $\mathrm{Al}_{\sim 70} \mathrm{Pd}_{\sim 21} \mathrm{Mo}_{\sim 1} \mathrm{Fe}_{\sim 8}$. Figure 6(a) shows high-resolution powder X-ray diffraction (HRPXRD) patterns obtained using synchrotron radiation (wave length: $\lambda=0.65298 \AA$, corresponding to $\mathrm{Nb} \mathrm{K}$ Abs. Edge) from single-phase polycrystalline samples prepared by annealing at three different temperatures. By comparing these and other measured HRPXRD patterns with a reference pattern published for the $\mathrm{Al}_{\sim 69} \mathrm{Pd}_{\sim 22} \mathrm{Cr}_{\sim 2} \mathrm{Fe}_{\sim 7}$ compound, ${ }^{27)}$ it has been confirmed that samples annealed at various temperatures within $1048-1233 \mathrm{~K}$ were isostructural to the $(2 \times 2 \times 2)$ cubic $3 / 2$ AP. However, the HRPXRD pattern of the sample annealed at $1023 \mathrm{~K}$, the lowest temperature tested, shows clear differences in the fine structure of peaks from those of the samples annealed at higher temperatures, indicating a structurally distinct phase (see Fig. 6(a)).
The two samples annealed at $1023 \mathrm{~K}$ and $1073 \mathrm{~K}$ were studied further under a scanning electron microscope (SEM: JXA-8621MX, JEOL) equipped with an electron probe micro-analyzer (EPMA). Figure 6(b) shows that SEM micrographs as well as the analyzed compositions of the two samples are practically indistinguishable, suggesting that there is a reversible structural phase transition at around $1030 \mathrm{~K}$ between the $(2 \times 2 \times 2)$ cubic $3 / 2 \mathrm{AP}$ and an unknown low-temperature phase.

In order to examine the crystal structure of the unknown phase, a single crystal sample was prepared using a two-step annealing process. In the first step, an ingot with a nominal composition of $\mathrm{Al}_{\sim 70} \mathrm{Pd}_{\sim 21} \mathrm{Mo}_{\sim 1} \mathrm{Fe}_{\sim 8}$ was initially melted at $1433 \mathrm{~K}$, slowly cooled down (by $5 \mathrm{~K} /$ hour) to $1198 \mathrm{~K}$, at which it was aged for 60 hours, cooled down (by $10 \mathrm{~K} /$ hour) to $998 \mathrm{~K}$, then brought down to room temperature by switching off the furnace. The resulting sample showed sub-millimeter sized shiny faceted crystal grains. Then, in the second step, the sample was re-annealed at $1023 \mathrm{~K}$ for 240 hours to ensure that the low-temperature phase would be obtained. SCXRD measurements were performed on tiny flakes taken from the sample, using a 4-axis X-ray diffractometer (MicroMax007 Varimax-DW with Saturn $724 C C D$, Rigaku) with Mo-K $\alpha$ radiation $(\lambda=0.71073 \AA)$.

The diffraction intensity distribution in the $3 \mathrm{D}$ reciprocal space from one of the flakes with the best quality was reconstructed using CrysAlis ${ }^{\text {Pro }}$ V.40 (Rigaku Oxford Diffraction, https://www.rigaku.com/products/smc/crysalis). As seen in selected 2D sections shown in Fig. 7(a), strong Bragg reflections could be indexed as a primitive cubic lattice with a lattice parameter of $20.24 \AA$. These can be interpreted 


\section{(a)}
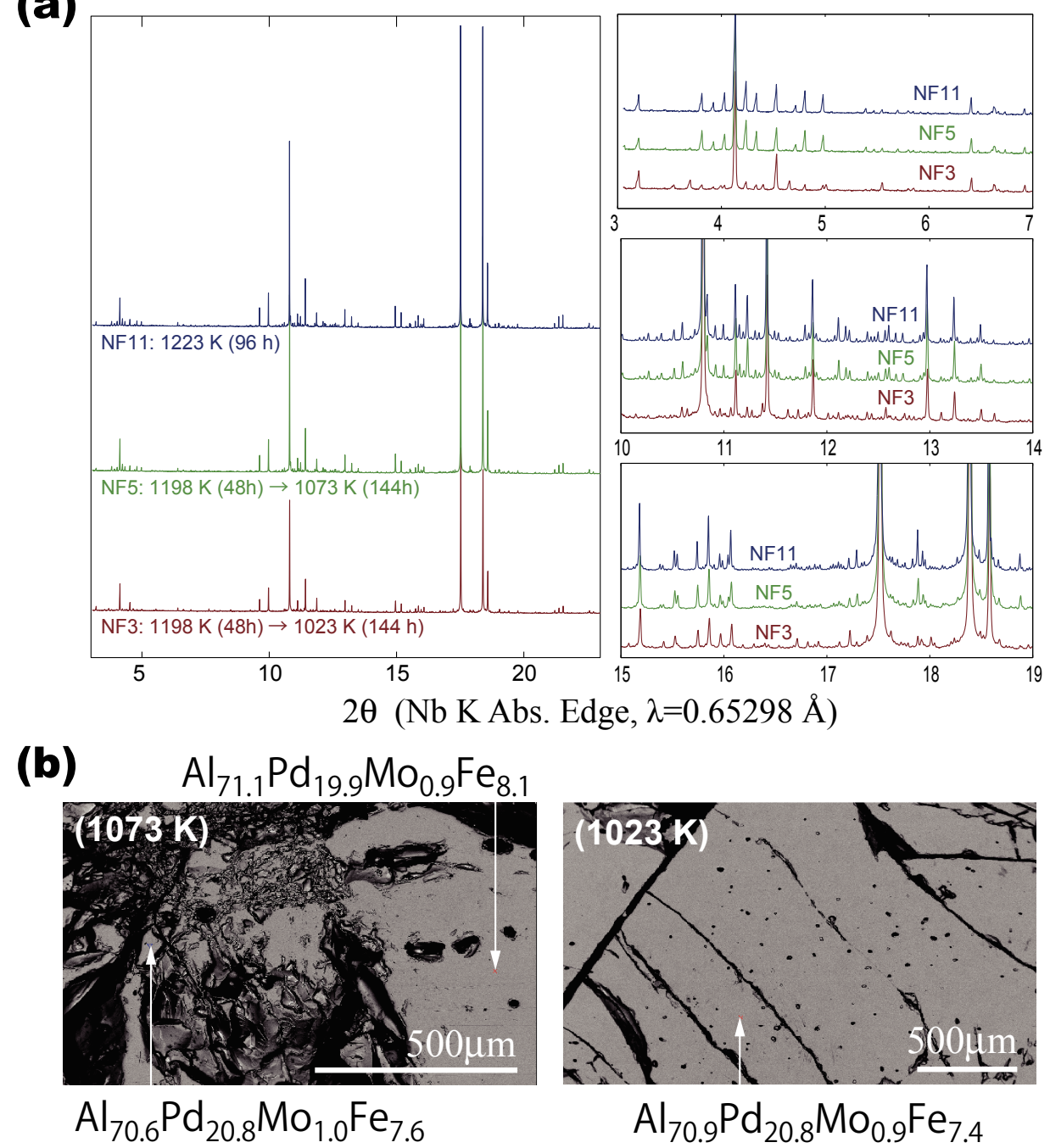

Fig. 6 (a) HRPXRD patterns obtained from single-phase polycrystalline samples of $\mathrm{Al}_{\sim 70} \mathrm{Pd}_{\sim 21} \mathrm{Mo}_{\sim 1} \mathrm{Fe}_{\sim 8}$ annealed at $1023 \mathrm{~K}$ (red), $1073 \mathrm{~K}$ (green) and $1223 \mathrm{~K}$ (blue), measured using synchrotron X-ray (wave length $=0.65298 \AA$ ). Magnified plots shown on the right reveal minute differences of the $1023 \mathrm{~K}$ sample from the rest. (b) SEM micrographs of the two samples annealed at $1073 \mathrm{~K}$ and $1023 \mathrm{~K}$. Analyzed compositions using EPMA at selected points in the images are also indicated.

as the fundamental reflections for the cubic $3 / 2$ AP. ${ }^{27)}$ Meanwhile, one also observes weak satellite reflections that can be exhausted by assuming a cubic supercell with 3-fold, instead of 2-fold, periodicity with a lattice parameter of $60.72 \AA$. However, as schematically illustrated in the left panel of Fig. 7(b), the satellite peaks exist only at the vertices of the 1/3-sized cubes co-centered with the cubes spanned by the fundamental reflections. This suggests that the present structure is better interpreted as twins of a trigonal superstructure of the cubic $3 / 2 \mathrm{AP}$, with the super-cell being 3 times as large in volume as the basic unit cell of the cubic $3 / 2$ AP (Fig. 7(b), right panel).

The structural complexity as well as the twinning feature makes an $a b$ initio structure determination of the latter trigonal AP using the SCXRD data overwhelmingly challenging, so that we haven't managed to complete the refinement yet. In the meantime, we are tempted to make a rational guess of the crystal structure by applying our geometrical framework.

In order to do so, we at first have to identify a CCT that underlies the crystal structure. In fact, a variety of candidate
CCTs, which are a super-structure of the cubic 3/2 packing with the specified trigonal super-cell, are possible. These can be efficiently generated using a similar technique to the so called point substitution process that led to the discovery of quasiperiodic CCTs. ${ }^{41)}$ One can postulate that the most relevant one would be the one with the largest number of vertices per unit cell, comprised of 48 even and 50 odd vertices (98 vertices in total).

Once the CCT is chosen, a hypothetical crystal structure can be readily constructed using the atomic decoration scheme of Sec. 4, as shown in the bottom row of Fig. 8(a). Here, we assumed that each atomic site is purely occupied by the characteristic element of the relevant orbit indicated by the superscript of the orbit symbol. A similar process for the $(2 \times 2 \times 2)$ cubic $3 / 2$ AP (the high-temperature phase) is illustrated in the top row of the same figure. The compositions of the two hypothetical structures are $\mathrm{Al}_{70.74} \mathrm{Pd}_{23.70} \mathrm{Fe}_{5.56}$ (high-temperature phase, $\mathrm{Pa} \overline{3}$ ) and $\mathrm{Al}_{70.47} \mathrm{Pd}_{23.57} \mathrm{Fe}_{5.96}$ (lowtemperature phase, $P 3$ ), which are practically identical.

Figure 8(b) shows calculated HRPXRD patterns of the two hypothetical structure models. Observe that the overall 
(a)
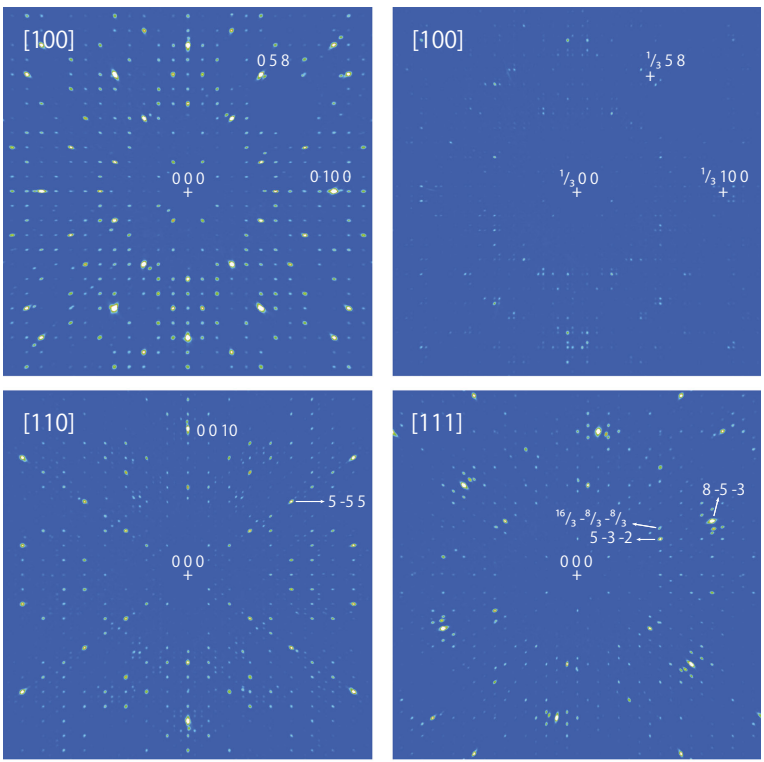

(b)
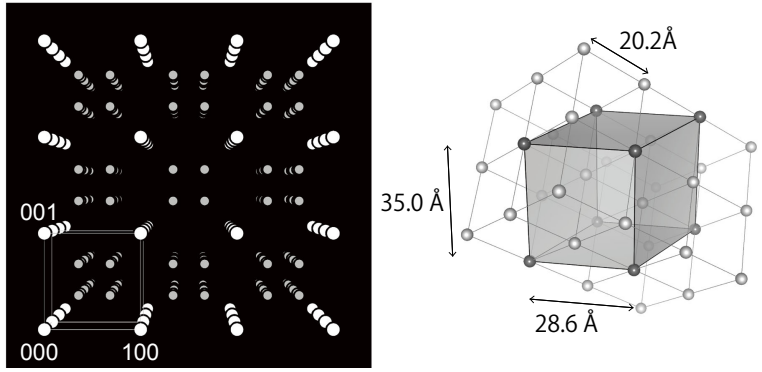

Fig. 7 (a) Selected 2D sections of the 3D reconstructed diffraction intensities obtained from a single crystal grain of the low temperature phase. Peaks are indexed with respect to the fundamental reflections representing a cubic lattice with a lattice parameter of $20.2 \AA$, corresponding to the cubic $3 / 2$ AP. Satellite peaks indexed as ( $h+\frac{h^{\prime}}{3}, k+\frac{k^{\prime}}{3}, l+\frac{l^{\prime}}{3}$ ) with $h^{\prime}, k^{\prime}$, and $l^{\prime}$ being 1 or 2 can be noticed. (b) Left panel: A schematic drawing of the observed satellite peak positions (smaller gray spheres) with respect to the fundamental peak positions (larger white spheres) corresponding to the cubic 3/2 AP. Right panel: A trigonal super-cell (colored in gray) that is 3 times as large in volume as the basic unit cell of the cubic $3 / 2 \mathrm{AP}$

features of the experimental HRPXRD patterns of the highand low-temperature phases of $\mathrm{Al}_{\sim 70} \mathrm{Pd}_{\sim 21} \mathrm{Mo}_{\sim 1} \mathrm{Fe}_{\sim 8}$ (Fig. 6(a)) are reproduced fairly well by the calculated patterns of the cubic and trigonal models, respectively. Noticeable discrepancies in the peak intensities can be attributed to oversimplifications in the calculations, such as the absence of chemical disorder and an identical value for the isotropic atomic displacement parameters of all the atoms. One may also notice that the experimental pattern of the 'low-temperature phase' indicates a small amount of residual 'high-temperature phase' (see, for example, double peaks near $2 \theta \simeq 4^{\circ}$ or $5^{\circ}$ in a magnified range of $3^{\circ}-7^{\circ}$ ).

\section{Conclusions}

In this paper, we have presented a unified geometrical framework to describe the atomic structure of a range of complex APs to FCI type QCs in Al-Pd-TM systems. The atomic structure is understood as a packing of pMCs and $\mathrm{mBCs}$ arranged on the even and odd vertices of a CCT, respectively. We have introduced 15 decoration orbits (a)

$\mathrm{Pa} \overline{3}$
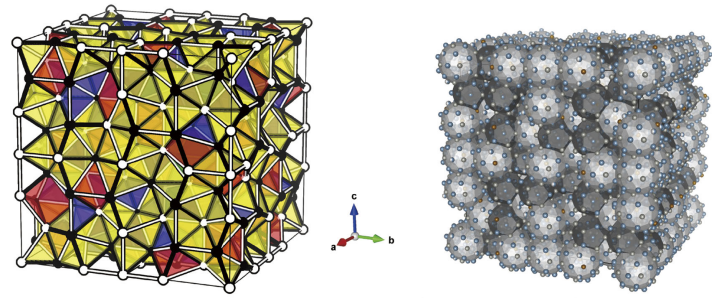

(b)
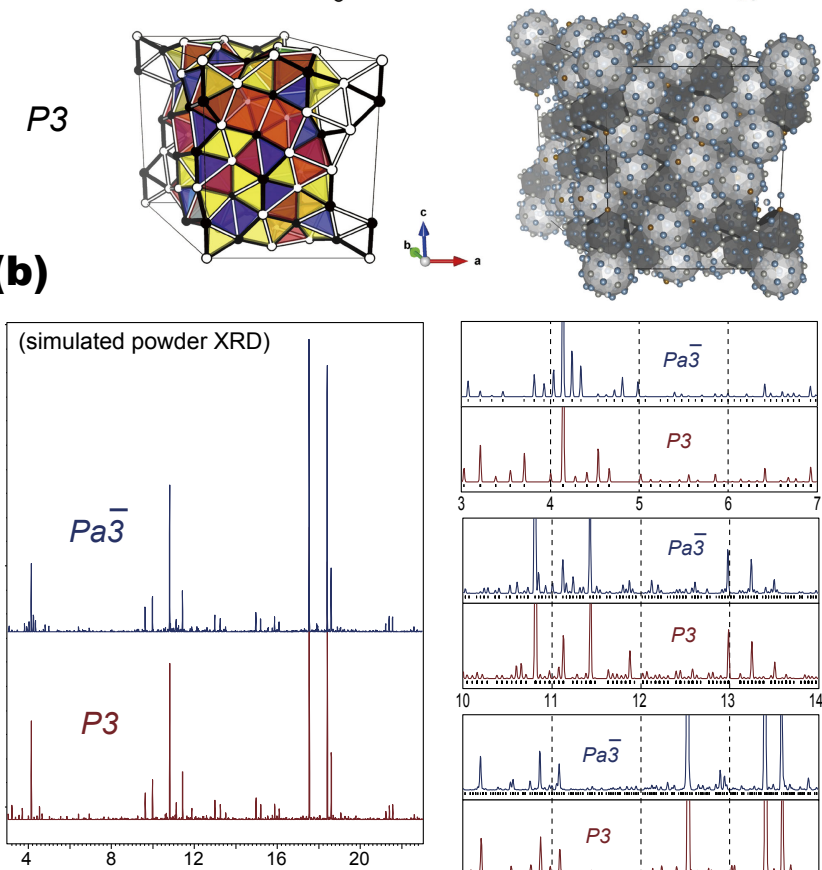

$2 \theta(\mathrm{Nb} \mathrm{K}$ Abs. Edge, $\lambda=0.65298 \AA)$

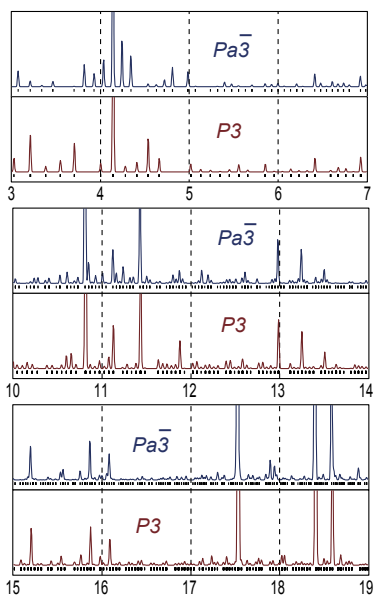

Fig. 8 (a) Two CCTs and their corresponding atomistic structure models obtained using the decoration scheme. The first row represents the $(2 \times 2 \times 2)$ cubic $3 / 2 \mathrm{AP}^{27}{ }^{27}$ The second row represents a trigonal superstructure of the $3 / 2$ AP. (b) Powder XRD patterns of the two structure models calculated using JANA2006 ${ }^{46)}$ with a wave length of $\lambda=$ $0.65298 \AA$. Magnified plots shown on the right reveal minute differences between the two.

associated with vertices, edges, faces, and cells in the CCT to register all the atomic positions. Importantly, atoms belonging to the same orbit are expected to possess similar characteristics in terms of chemical species as well as thermal vibrations due to their similar local environment.

We have also demonstrated the use of the present geometrical framework to predict an unknown crystal structure of a novel AP phase in the Al-Pd-(Mo-Fe) system. We could further foresee a potential merit of the present framework in its ability to make a rational guess of a complex AP structure, even if an ab initio structure determination is hindered by the complexity itself, intricate features like twinning, or even insufficient quality of data and samples. Our decoration scheme would provide a starting model for the structure refinement if a likely candidate of the underlying CCT could be postulated. This would pave the way to elucidate a number of novel AP structures with large unit cells as well as intricate twinning features.

\section{Acknowledgments}

The authors are deeply indebted to An-Pang Tsai for his support and encouragement while working on the present 
subject. They also gratefully acknowledge Satoshi Ohhashi, Akira Sato, Yoshitaka Matsushita, Masahiko Tanaka, Yoshio Katsuya for their technical supports with SEM and X-ray diffraction. N.F. thanks Marek Mihalkovič for helpful discussions on the CCT framework and atomic decoration schemes. HRPXRD experiments using synchrotron radiation were performed at the BL15XU beamline of SPring-8 under the approval of National Institute for Materials Science (NIMS) (Proposal Nos. 2015A4901, 2016A4900, and 2017A4901). SCXRD experiments were done under the support of NIMS microstructural characterization platform as a program of "Nanotechnology Platform" of the Ministry of Education, Culture, Sports, Science and Technology (MEXT), Japan. The VESTA (Ver. 3) package ${ }^{45)}$ was extensively used for the graphical presentation of structures. This work was financially supported by Japan Science and Technology Agency through PRESTO (Grant No. JPMJPR1519) and Japan Society for the Promotion of Science through Grant-in-Aid for Scientific Research (Grant Nos. 25400354, 18K04677 and JP19H05819).

\section{REFERENCES}

1) D. Shechtman, I. Blech, D. Gratias and J.W. Cahn: Phys. Rev. Lett. 53 (1984) 1951-1953.

2) A.-P. Tsai: Sci. Technol. Adv. Mater. 9 (2008) 013008.

3) A.-P. Tsai: Chem. Soc. Rev. 42 (2013) 5352-5365.

4) H. Takakura, C.P. Gómez, A. Yamamoto, M. de Boissieu and A.-P. Tsai: Nat. Mater. 6 (2007) 58-63.

5) A. Yamamoto, H. Takakura and A.-P. Tsai: Phys. Rev. B 68 (2003) 094201.

6) J. Dolinšek: Chem. Soc. Rev. 41 (2012) 6730-6744.

7) A.-P. Tsai, A. Inoue and T. Masumoto: Jpn. J. Appl. Phys. 26 (1987) L1505-L1507.

8) A.-P. Tsai, A. Inoue and T. Masumoto: Jpn. J. Appl. Phys. 27 (1988) L1587-L1590.

9) A.-P. Tsai, Y. Yokoyama, A. Inoue and T. Masumoto: Jpn. J. Appl. Phys. 29 (1990) L1161-L1164.

10) T. Shibuya, T. Asao, M. Tamura, R. Tamura and S. Takeuchi: Mater. Sci. Eng. A 294-296 (2000) 61-64.

11) T. Asao, R. Tamura and S. Takeuchi: Philos. Mag. Lett. 82 (2002) $217-$ 223.

12) E.M. Barber: Applied Sciences 9 (2019) 2132.

13) J.-M. Dubois: Isr. J. Chem. 51 (2011) 1168-1175.

14) J.-M. Dubois: Chem. Soc. Rev. 41 (2012) 6760-6777.

15) P. Bak: Phys. Rev. Lett. 56 (1986) 861-864.
16) A. Yamamoto: Acta Crystallogr. Sec. A 52 (1996) 509-560.

17) D.S. Rokhsar, N.D. Mermin and D.C. Wright: Phys. Rev. B 35 (1987) 5487-5495.

18) L.S. Levitov and J. Rhyner: J. Phys. France 49 (1988) 1835-1849.

19) The three Bravais classes can also be obtained through a symmetrical reduction of the simple, face-centered and body-centered hyper-cubic lattices in six dimensions.

20) A.I. Goldman and R.F. Kelton: Rev. Mod. Phys. 65 (1993) 213-230.

21) W. Steurer and S. Deloudi: Acta Crystallogr. Sec. A 64 (2008) 1-11.

22) T. Yamada, H. Takakura, M. de Boissieu and A.-P. Tsai: Acta Crystallogr. Sec. B 73 (2017) 1125-1141.

23) T. Yamada, H. Takakura, T. Kong, P. Das, W.T. Jayasekara, A. Kreyssig, G. Beutier, P.C. Canfield, M. de Boissieu and A.I. Goldman: Phys. Rev. B 94 (2016) 060103(R).

24) T. Yamada, H. Takakura, H. Euchner, C.P. Gómez, A. Bosak, P. Fertey and M. de Boissieu: IUCrJ 3 (2016) 247-258.

25) C.L. Henley: Phys. Rev. B 43 (1991) 993-1020.

26) G. Kreiner: J. Alloy. Compd. 338 (2002) 261-273.

27) N. Fujita, H. Takano, A. Yamamoto and A.-P. Tsai: Acta Crystallogr. Sec. A 69 (2013) 322-340.

28) Y. Hatakeyama, N. Fujita and A.-P. Tsai: J. Phys.: Conf. Ser. 809 (2017) 012007.

29) K. Sugiyama, K. Yubuta, Y. Yokoyama, S. Suzuki and R. Simura: Acta Phys. Pol. A 126 (2014) 588-593.

30) R. Simura, S. Suzuki, K. Yubuta and K. Sugiyama: Mater. Trans. 54 (2013) 1385-1391.

31) A. Katz and D. Gratias: J. Non-Cryst. Solids 153-154 (1993) 187-195.

32) D. Gratias, M. Quinquandon and A. Katz: Quasicrystals, Current Topics, (World Scientific, Singapore, 2000) pp. 1-72.

33) V. Elser: Philos. Mag. B 73 (1996) 641-656.

34) P. Kramer and R. Neri: Acta Crystallogr. Sec. A 40 (1984) 580-587.

35) M. Duneau and A. Katz: Phys. Rev. Lett. 54 (1985) 2688-2691.

36) A. Katz: Commun. Math. Phys. 118 (1988) 263-288.

37) A.L. Mackay: Physica A 114 (1982) 609-613.

38) K. Sugiyama, N. Kaji, K. Hiraga and T. Ishimasa: Z. Kristallogr. 213 (1998) 90-95.

39) K. Sugiyama, N. Kaji, K. Hiraga and T. Ishimasa: Z. Kristallogr. 213 (1998) 168-173.

40) K. Toyonaga, R. Shibata, T. Yamada, M. de Boissieu, O. Peréz, P. Fertey and H. Takakura: Philos. Mag. 100 (2020) 2220-2243.

41) N. Fujita: Ann. Phys. 385 (2017) 225-286.

42) A.-P. Tsai, T. Masumoto and A. Yamamoto: Philos. Mag. Lett. 66 (1992) 203-208.

43) A.-P. Tsai, A. Inoue and T. Masumoto: Mater. Trans. JIM 34 (1993) $155-161$.

44) M. Mihalkovič, W.-J. Zhu, C.L. Henley and M. Oxborrow: Phys. Rev. B 53 (1996) 9002-9020.

45) K. Momma and F. Izumi: J. Appl. Cryst. 44 (2011) 1272-1276.

46) V. Petř́íček, M. Dušek and L. Palatinus: Z. Kristallogr. 229 (2014) 345352. 